\title{
Grid Information Service Based on Network Hops*
}

\author{
Xia Xie, Hai Jin, Jin Huang, and Qin Zhang \\ Cluster and Grid Computing Lab \\ Huazhong University of Science and Technology, Wuhan, 430074, China \\ hjinahust.edu.cn
}

\begin{abstract}
Grid information service influences outcome of applications on grid platforms directly. In this paper, network coordinate is introduced in grid information service mechanism to locate each grid node. With the hop count generation algorithm, network hops between user and resource providers can be forecasted, and results can be submitted to grid information service, which offers a list of resource providers with network hops increasing so that scheduler can work more efficiently. Performance proves that it is suitable for timesensitive applications or applications with special restriction of network hops.
\end{abstract}

\section{Introduction}

Grid system can offer several key services and one of them is Grid Information Service (GIS) [4]. Relational Grid Monitoring Architecture (R-GMA) offers a global view of the information [1]. Globus Toolkit's Monitoring and Discovery System (MDS) defines and implements mechanisms for service and resource discovery and monitoring in distributed environments. The latest version MDS4 [6] is defined in the new WS-Resource Framework and WS-Notification specifications.

In this paper, GIS based on network hops is proposed. By using the network hops, the network distance between the user and resource providers can be calculate. A list of candidate resource providers with hop count in increasing order can be returned automatically. User can choose the resource provider with minimum network hops.

\section{Design Principle}

We predict the hop count between two nodes according to their network coordinates. Some notations are defined as follows. $L_{i j}$ stands for actual hop counts between node $i$ and node $j . X_{i}$ is the network coordinates of node $i . E$ is the system squared-error. $L$ is an aggregate of nodes and the sum of nodes is Listlength $(\mathrm{L}) . d$ is a constant defined according to user's requirement.

$$
E=\sum_{i} \sum_{j}\left(L_{i j}-\left\|x_{i}-x_{j}\right\|\right)^{2}
$$

This paper is supported by National Science Foundation of China under grant 60125208 and 90412010, ChinaGrid project of Ministry of Education of China. 
$\left\|x_{i}-x_{j}\right\|$ is the distance of network coordinates from node $i$ to $j$ in appointed coordinate space. Hop count algorithm calculates the unit-length vector from node $i$ to $j$ by minimizing Eq.1. In order to reduce the network traffic, we change the policy that each node running hop count algorithm only communicates with part of the other nodes. These parts of the other nodes make up of an aggregate $L$ and the nodes in $L$ must not less than $d+1$.

Hop count algorithm generates a unit-length vector in randomly chosen direction and detaches two nodes at a same location so that network coordinate of each node can be quickly convergent. The node running the hop counts algorithm keeps on updating network coordinates periodically. If the network topology changes, node will update automatically. With the network coordinates of node $i$ and $j$, the third information service node can forecast the network hops between these two nodes though node $i$ does not communicate or measure the actual hop count with node $j$.

For hierarchical information service, node can get its network coordinates by running the network hop count algorithm. New user node or new resource provider node gets the IP address, network coordinates and estimated error of other nodes through the information providers and composes of its own $L$. For information service in the P2P style, each node runs the hop count algorithm to get its own network coordinates. New node gets the IP address, network coordinates and estimated error of other nodes through an arbitrary node to make up of $L$.

Index service is embedded with an optimizer of network hop count. Function of the optimizer is to calculate the hop count between user and candidate resource provider through their network coordinates and sort the hop count in increasing order. Index service returns a list of candidate resource providers to users but not ensures the availability of each resource provider. Index service also does not include policy information and resource provider need not to publish its own policy. For user who does not select the attached service, user can negotiate with each result item one by one. Only after authenticated can the resource provider deal with the submitted task.

\section{Experiments and Analysis}

Two performance metrics are used here: average response time and throughput of information discovery. Average response time of information discovery is the time from information discovery requirement being sent out to the time the results return. It is an average value for multiple simulations. We call the general information service without optimizer "random information service".

We use a grid simulation, JFreeSim [2]. JFreeSim is a grid simulation tool based on multiple tasks, multiple schedulers and multiple resources model. As a modular and extensible simulation tool, JFreeSim realizes many entity modeling and communication mechanisms between all entities, and makes system simulation according with the characteristics of the grid environment.

We use the Inet [3] topology generator to create 8,000-node wide-area AS-level network with a variable number of 500 client nodes with 4 client nodes per stub. ModelNet [5] is used to emulate wide-area bandwidth and latencies. Transit-transit and transit-stub links are $155 \mathrm{Mb} / \mathrm{s}$ and client-stub links are $2 \mathrm{Mb} / \mathrm{s}$. Latencies are based on the Inet topology. The 500 client nodes are simulated by 11 PCs running 
JFreeSim. Hop count algorithm is implemented in each information entity, grid user entity or resource entity generated by JFreeSim. Each PC has $1.6 \mathrm{GHz}$ Pentium IV processor and $512 \mathrm{MB}$ RAM with Redhat 9.0 operating system. All PCs are connected using Gigabit Ethernet. One of 11 PCs simulates information service and information service uses MDS4. The number of resource providers is 50, 100, 200, and 400 , respectively. Other $10 \mathrm{PCs}$ are used to simulate resource providers and users. The number of simulated grid user is $80,160,240,320,400$, and 480 , respectively.

Fig. 1 describes the average response time of information discovery for random information service and GIS based on network hops. For the later, it is also divided into two types: one is resource discovery with the range of hops, which is less than 10 and submitted by users; another is resource discovery without giving the range of network

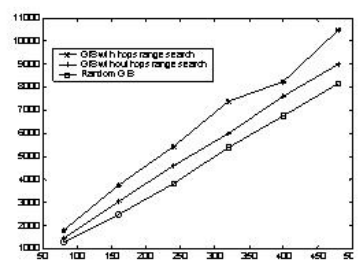

The number of resources is 50 .

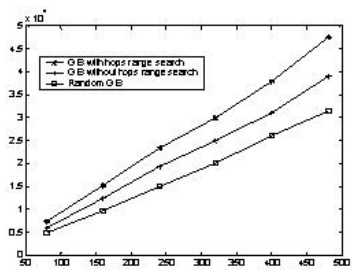

The number of resources is 200

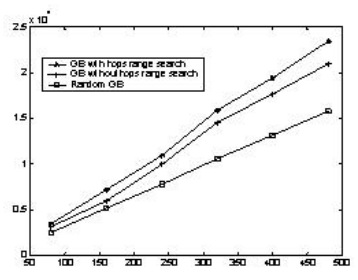

The number of resources is 100 .

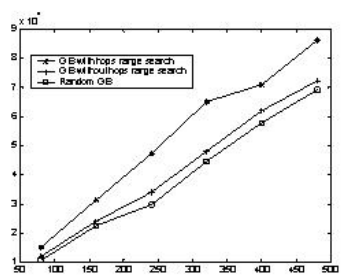

The number of resources is 400 .

Fig. 1. Average response time for information discovery
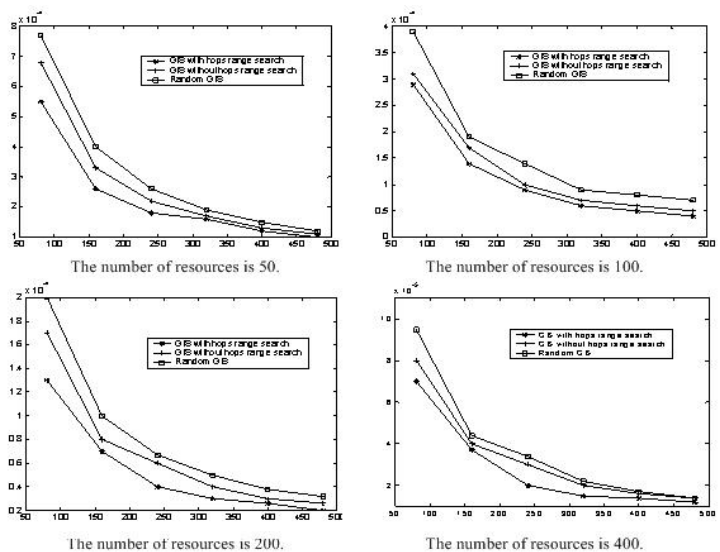

The number of resources is 400 ,

Fig. 2. Throughput for information discovery 
hops. Comparing these figures, we can draw conclusions as follows: 1) GIS based on network hops has longer average response time; 2) information discovery with the range of hops has longer average response time than information discovery without giving the range of network hops; 3 ) more information provided by resource providers, more average response time.

Fig. 2 describe throughput of information discovery. We find: 1) throughput of information discovery in GIS based on network hops is smaller than random information service; 2) more resource providers, less throughput; 3) information discovery with the range of hops has smaller throughput than information discovery without giving the range of network hops.

\section{Conclusions}

In order to improve performance of the grid information service in time-sensitive application, we propose a grid information service based on network hops. Using network coordinate, the network hops between user and candidate resource provider can be predicted, and a list of network hops in increasing order can be returned. User can use the resource provider with minimum network hops. The grid resource sharing and cooperation can be more efficiently.

\section{References}

1. A. W. Cooke, A. J. G. Gray, L. Ma, W. Nutt, J. Magowan, M. Oevers, P. Taylor, R. Byrom, L. Field, S. Hicks, J. Leake, M. Soni, A. J. Wilson, R. Cordenonsi, L. Cornwall, A. Djaoui, S. Fisher, N. Podhorszki, B. A. Coghlan, S. Kenny, and D. O'Callaghan, "R-GMA: An Information Integration System for Grid Monitoring", Proceeding of the $10^{\text {th }}$ International Conference on Cooperative Information System, 2003.

2. H. Jin, J. Huang, X. Xie, and Q. Zhang, "JFreeSim: A Grid Simulation Tool Based on MTMSMR Model", Proceedings of 6th International Workshop on Advanced Parallel Processing Technologies, Hong Kong, China, 2005.

3. H. Chang, R. Govindan, S. Jamin, S. Shenker, and W. Willinger, "Towards Capturing Representative AS-level Internet Topologies", Int. J. Comp. and Tele. Net. 44 (2004), pp.737755.

4. H. N. L. C. Keung, J. R. D. Dyson, S. A. Jarvis and G. R. Nudd, "Performance Evaluation of a Grid Resource Monitoring and Discovery Service", IEE Proceeding on Software, 150, 2003, pp.243-251.

5. K. Yocum, K. Walsh, A. Vahdat, P. Mahadevan, D. Kostic, J. Chase, D. Becker, "Scalability and Accuracy in A Large-Scale Network Emulator", Proceeding of the Fifth Symposium on Operating Systems Design and Implementation, 2002, pp.271-284.

6. J. M. Schopf, M. D'Arcy, N. Miller, L. Pearlman, I. Foster, and C. Kesselman, "Monitoring and Discovery in A Web Services Framework: Functionality and Performance of the Globus Toolkit's MDS4", Technical report, printed by ANL/MCS, 2004, pp.1248-1260. 